\title{
Optimal Consumption and Labor Choices with Learning-by-Doing
}

\author{
Marina Di Giacinto1, Francesco Ferrante ${ }^{1,2}$ \\ ${ }^{1}$ Dipartimento di Economia e Giurisprudenza, Università degli studi di Cassino e del Lazio Meridionale, Cassino, Italy \\ ${ }^{2}$ Dipartimento di Scienze Politiche, LUISS Guido Carli, Roma, Italy \\ Email: digiacinto@unicas.it,f.ferrante@unicas.it
}

How to cite this paper: Di Giacinto, M., \& Ferrante, F. (2021). Optimal Consumption and Labor Choices with Learning-by-Doing. Theoretical Economics Letters, 11, 1221-1246. https://doi.org/10.4236/tel.2021.116078

Received: October 17, 2021

Accepted: December 24, 2021

Published: December 27, 2021

Copyright (c) 2021 by author(s) and Scientific Research Publishing Inc. This work is licensed under the Creative Commons Attribution International License (CC BY 4.0).

http://creativecommons.org/licenses/by/4.0/

\begin{abstract}
The net accumulation of human capital through the cultivation of cognitive and non-cognitive skills and knowledge is an important source of change in professional and personal peoples' life. Past work experience has a direct effect on wage levels and their growth; thus, it influences the achievable level of consumption and leisure and lifecycle wealth. This paper analyzes the relation between labor supply, consumption, and human capital accumulation in a dynamic learning-by-doing setting. We model in a continuous-time framework, the optimal control problem of a household that takes decisions on the level of consumption and labor supplied to maximize logarithmic utility from consumption and leisure subject to the constraints arising from the intertemporal accumulation of human and liquid wealth. We analyze an optimal learning-by-doing program in a deterministic framework, determine explicitly the optimal control policies, and comment on their economic implications.
\end{abstract}

\section{Keywords}

Human Capital, Learning-by-Doing, Consumption, Labor Supply, Dynamic Programming

\section{Introduction}

Labor markets are nowadays experiencing fast and structural transformations. Skill and educational mismatches are evident signals of the effects of this faster pace of change (OECD, 2018). It is a documented fact (Acemoglu \& Autor, 2011; Autor \& Dorn, 2013; Autor, Levy, \& Murnane, 2003; D’Orlando, Ferrante, \& Oliverio, 2021) ${ }^{1}$ that these transformations are relevant drivers also of the rising

${ }^{1}$ D'Orlando, Ferrante, and Oliverio (2021) offer an overview of the effect of transformations of the labor market brought about by globalization and the ICTs. 
levels of inequality. Even though inequality starts early in life, the empirical analysis has highlighted striking differences in earnings growth over the lifecycle (see, e.g., Karahan, Ozkan, \& Song, 2019). The ability to accumulate human capital and to raise compensation per worked hour depends on many different factors that certainly include innate abilities but are often more interconnected with the career paths and the possibility to improve personal abilities beyond the level achieved during the schooling period and in connection with the educational attainment.

The international surveys on the learning outcomes show that test scores in basic skills reach the maximum around 30 years and that thereafter we experience their depletion. So, after that age, the net accumulation of human capital depends a lot on people's exposure to effective learning mechanisms (OECD, 2013).

The net accumulation of human capital, i.e., the cultivation of cognitive and non-cognitive skills and of knowledge, is the main source of change in people's professional and personal life. It determines our effectiveness in decision making as well as our opportunities in different life domains ${ }^{2}$. The pace of accumulation depends on learning opportunities available in the environment we live in, in particular, from the opportunity to cultivate our skills in the labour market through formal training and experience.

There is strong evidence that the process of human capital accumulation is determined in a crucial way by people's investments in education early in life and that our opportunity to learn over time is positively associated with our educational attainment (Brunello, Garibaldi, \& Wasmer, 2007). The opportunity to cultivate our skills is also affected by the type of education: according to empirical evidence and theoretical speculations, general education is more effective than vocational or specific education in supporting the workers' adaptation to labour market needs (see Hanushek et al., 2017).

The crucial role and the riskiness of the investment in education early in life is mirrored by the empirical evidence on what adult people regret most in life showing that educational and career choices are the most important sources of regret (e.g., should have stayed in school, should have studied harder, should have got another degree). RoeSum provides a meta-analysis based on surveys on life regrets carried out between 1989 and 2003 summarized in Table 1. They suggest that educational choices are affected by serious informative failures and that the latter is overcome only when people enter the labour market. However, it is not the aim of this paper to afford the analysis of the factors behind regrets.

It is noteworthy that the locus of connection between the two main sources of regret, i.e., educational and carrier choices, is the labour market.

${ }^{2}$ Human Human capital is an essential input in both production and consumption activities. It includes innate personality traits as well as abilities/skills acquired through education and experience. Building on Heckman, Stixrud, and Urzua (2006), Chuna and Heckman (2007) argue that the productivity of human capital (i.e., human capital in efficiency units) in both consumption and work activities depends on the actual matching between people's endowment of human capital and the characteristics of, respectively, the available jobs and consumption goods. 
Table 1. What we regret most in life.

\begin{tabular}{ccc}
\hline Area & $\%$ \\
\hline Education & 32.2 \\
Career & 22.3 \\
Romance & 14.8 \\
Being parents & 10.2 \\
Self & 5.5 \\
Leisure & 2.5 \\
Finance & 2.5 \\
Family & 2.3 \\
Health & 1.5 \\
Friends & 1.5 \\
Other & 4.7 \\
\hline
\end{tabular}

Source: Roese and Summerville (2005).

Within fast-changing environments, such as those we are facing due to globalization and the ICT revolution, the need to cultivate skills becomes crucial and this necessity is even more important if we consider that, due to the increase in life expectancy, the skills will be needed over a more extended life span. In this context, there are at least two topics that deserve to be afforded on theoretical and empirical grounds:

1) Labour market and consumption choices with human capital accumulation;

2) The interactions between the investment in education early life and the accumulation of human capital over life.

This paper deals with both issues in a novel way by providing a closed solution to the underlying analytical problem. We develop a model of human capital accumulation by assuming that the pace of accumulation is determined by the investment in education early in life jointly with labour and consumption choices over the working life. Our analytical framework help explain the connections between learning regimes and inequality in wellbeing from a lifecycle perspective. In particular, we point at how career discontinuity, due to frequent unemployment episodes, or anticipated skills obsolescence, due to fast technical change, can adversely affect less-educated workers and workers with more specific skills.

The first studies about human capital have begun with the works done by Schultz (1963) and Becker (1964). In a neoclassical theory of growth, the role of human capital was highlighted, to our knowledge, by Uzawa (1965), and much more lately by Lucas (1988) and Romer (1990).

With the emergence of the "new" growth theory, human capital accumulation 
and its role in economic growth has become a major area of research in macroeconomics. The literature starts with the seminal paper of Lucas (1988) which shows that the growth rate of per capita income depends on the growth rate of human capital which again depends on the time allocation of the individuals for acquiring skill. Lucas (1988) considers the prospects for constructing a neoclassical theory of growth and international trade that is consistent with some of the main features of economic development.

Since then, many economists have dealt with the issue of human capital accumulation and growth and the Lucas (1988) model has been extended in various directions.

Blandin and Peterman (2019) discuss a variety of ways that the macroeconomic literature incorporates human capital accumulation. Three human capital technologies are common in the macroeconomic literature: exogenous accumulation, learning-by-doing, and learning-or-doing. Under exogenous human capital accumulation, workers take their lifecycle human capital profiles as given. Under learning-by-doing, an agent acquires human capital endogenously by working, which means they learn and earn labor income simultaneously. Under learning-or-doing, which is also referred to as Becker-Ben Porath skill accumulation or on-the-job training, workers accumulate human capital by spending some of their non-leisure time training. Therefore, learning-or-doing learning and producing are separate, mutually exclusive activities.

As many economists have observed, on-the-job training or learning-by-doing appear to be at least as important as schooling in the formation of human capital.

Cossa, Heckman, and Lochner (2002) observe that the crucial economic difference between the learning-or-doing model and the learning-by-doing model of skill formation is not that one model excludes leisure while the other includes it. The major source of the difference is that in the learning-or-doing model, investment and labor supply compete for the agent's time budget while in the learning-by-doing model they do not. In the learning-or-doing model, higher investment comes at the cost of foregone earnings. In the conventional learning-by-doing model, work and investment are the same activity. A worker receives more current and future earnings by working more today. There is no cost of foregone earnings for investment. The only cost is foregone leisure.

Imai and Keane (2004) solve and estimate a dynamic model that allows agents to optimally choose their labor hours and consumption and that allows for both human capital accumulation and savings. Estimation results and simulation exercises indicate that the intertemporal elasticity of substitution is much higher than the conventional estimates and the downward bias comes from the omission of the human capital accumulation effect. The human capital accumulation effect renders the life-cycle path of the shadow wage relatively flat, even though wages increase with age. Hence, a rather flat life-cycle labor supply path can be reconciled with high intertemporal elasticity of substitution.

The approach we are going to consider will focus on the "mechanics" of the 
learning-by-doing. There are two kinds of capital in the system: financial capital whose rate of accumulation depends on a risk-free rate of return and on the wage compensation of labor, and human capital whose rate of accumulation depends on innate abilities and on the amount of labour. The agent derives her utility from both consumptions out of financial capital and from leisure.

We employ a human capital accumulation rate close to the one used by Chang, Gomes, and Schorfheide (2002). We take into consideration a finite time horizon while Chang, Gomes, and Schorfheide (2002) focus their analysis of learning-by-doing in an infinite horizon business cycle model.

In learning-by-doing models, past work experience has a direct effect on the determination of wage and influences the achievable level of consumption and leisure.

The learning-by-doing model postulates that wages grow with experience: workers do not decide whether or not to invest in human capital, the simple state of being in employment generates returns in its own right. The wage depends positively on experience capital, and experience capital, in turn, depends positively on past participation. This implies that work not only brings immediate returns but also increases future wages by adding to experience. In this paper, we aim to explore in a simple model how different determinants may affect the ability of households to maximize their utility by properly selecting career paths and level of effort put in the daily working life.

We consider a lifecycle model with endogenous human capital accumulation. There are two types of capital in our model: liquid and human. Liquid wealth is accumulated during the lifecycle investing at a constant rate of return. Agents begin their work life with a small positive liquid capital and leave intentional bequests at the end of their life. On the other hand, agents start their work life with positive human capital, and human capital is accumulated only by allocating time to work by learning-by-doing.

In this framework, we naturally model an optimal control problem in a continuous-time and finite horizon where the state equation is the dynamics of both human capital and wealth, and the control is composed of both consumption and labor supply. Considering a logarithmic utility depending on current consumption and labor, and applying the tools of the dynamic programming (for a general reference see, e.g., Bardi \& Capuzzo-Dolcetta, 1997, and Yong \& Zhou, 1999), we analyze the optimal policies for the utility maximizer over the lifecycle showing that the human capital induces substantial change in people's labor and consumption choices.

To the best of our knowledge, this is the first time that a model incorporating household consumption and labor supply decisions with the accumulation of the human capital has been solved in a closed form. The solution has been found in a deterministic setting and takes into account some natural constraints (on consumption and on labor supply). Certainly, this is the first step for further research.

The paper is organized as follows. Section 2 develops the model of human 
capital accumulation and defines the intertemporal utility functional. Section 3 is devoted to set up the control problem and the dynamic programming approach solving analytically the resulting Hamilton-Jacobi-Bellman equation for a logarithmic utility function, finding the value function and the optimal policies through a verification argument. In Section 4 we give an analysis of the optimal strategies. Section 5 draws the main conclusions and further research. All the proofs are placed in Appendix.

\section{Notation}

In what follows we will denote $C^{1}(\mathbb{T} ; \mathbb{R})$ the set of all real-valued differentiable functions $f: \mathbb{T} \rightarrow \mathbb{R}, \mathbb{T}:=[0, T]$, and $\mathbb{T}^{-}:=[0, T), T \in(0,+\infty)$, $\Gamma:=(0,+\infty) \times[0,1), \quad \Lambda:=(0,+\infty) \times(0,+\infty)$.

\section{The Model}

We consider in a continuous-time framework a single representative agent who chooses how to allocate consumption and labor over the lifecycle $\mathbb{T}$. She can decide the amount of present and future consumption and the amount of work and leisure. Consumption can be differed by investing the present liquid wealth at a constant instantaneous rate of return $r>0$. Therefore the differential equation that describes the dynamics of liquid wealth is the following

$$
\left\{\begin{array}{l}
\mathrm{d} x(t)=[w(t) \ell(t)-c(t)+r x(t)] \mathrm{d} t, \quad t \in \mathbb{T}, \\
x(0)=x_{0}>0,
\end{array}\right.
$$

where

(H1) $\quad w: \mathbb{T} \rightarrow(0,+\infty)$ is the instantaneous (nominal) wage rate;

$(\mathrm{H} 2) \quad \ell: \mathbb{T} \rightarrow[0,1)$, is a measurable function that denotes the normalized amount the representative agent works;

(H3) $\quad c: \mathbb{T} \rightarrow(0,+\infty)$ is a measurable function that represents the consumption rate;

Following, e.g., MaCurdy (1999) and Shaw (1989), we suppose that the wage depends on the representative agent's human capital according to the relation

$$
w(t)=\theta h(t),
$$

where $h: \mathbb{T} \rightarrow(0,+\infty)$ represents the human capital while the rental rate $\theta>0$, is the market price of the services of a unit of human capital. It is the market-clearing price at which the aggregate supply and aggregate demand for human capital services are in equilibrium and in our setting we assume that it is exogenous and time-independent.

The stock of human capital of each worker is described by the following differential equation

$$
\left\{\begin{array}{l}
\mathrm{d} h(t)=[\lambda+\mu \ell(t)] h(t) \mathrm{d} t, \quad t \in \mathbb{T} \\
h(0)=h_{0}>0
\end{array}\right.
$$


where the growing rates of the human capital $\lambda, \mu \geq 0$ are hypothesized to be constant. In the context of a human capital accumulation model, the parameters $\lambda$ and $\mu$ have particularly interesting interpretations: both relate to the endowed ability of the individual to produce human capital in a way that is not dissimilar to what psychologists are attempting to measure. While $\lambda$ represents the rate of growth of human capital that cannot be improved by increasing the working effort, $\mu$ identifies the marginal increase in the human capital growth rates per unit of worked hour.

As a class of admissible controls, we consider the pairs of functions $(c(\cdot), \ell(\cdot))$ satisfying $(\mathrm{H} 2)-(\mathrm{H} 3)$ above and such that the corresponding dynamics of liquid wealth is nonnegative, i.e., no-bankruptcy constraint is satisfied.

Observe that state Equations (1)-(3) have a unique solution for any given admissible control $(c(\cdot), \ell(\cdot))$.

The optimization problem consists in maximizing over the set of the admissible controls $(c(\cdot), \ell(\cdot))$ the objective utility functional associated with the above state Equations (1)-(3)

$$
J(c(\cdot), \ell(\cdot))=\int_{0}^{T} \mathrm{e}^{-\rho t} u(c(t), \ell(t)) \mathrm{d} t+\mathrm{e}^{-\rho T} b(x(T), h(T)),
$$

where $x(\cdot):=x(\cdot ; c(\cdot), \ell(\cdot)), h(\cdot):=h(\cdot ; c(\cdot), \ell(\cdot))$ are the unique solutions to (1), (3), under the control $(c(\cdot), \ell(\cdot))$, respectively. Moreover:

(H4) $\rho>0$ is the subjective intertemporal discount factor;

(H5) $u: \Gamma \rightarrow \mathbb{R}$ is a sublinear growth function that represents the instantaneous utility function of the representative agent;

(H6) $b: \Lambda \rightarrow \mathbb{R}$ is the bequest function with a sublinear growth.

Notice that under the above hypotheses functional (4) is well-defined.

\section{Dynamic Programming}

Note that the initial time $t=0$ and the initial state $x(0)=x_{0}$ and $h(0)=h_{0}$ are fixed in the previous formulation. However, the basic idea of the dynamic programming (DP hereafter) technique is to consider a family of optimal controls problems with different initial times and states, establish a relationship among these problems, and finally solve all of them. Therefore to study the problem by the tool of the DP we define the problem for generic initial data $(t, x, h) \in \mathbb{T}^{-} \times \Lambda$, and consider the state variable whose dynamics are given by

$$
\left\{\begin{array}{l}
\mathrm{d} x(s)=[w(s) \ell(s)-c(s)+r x(s)] \mathrm{d} s, \quad s \in[t, T], \\
\mathrm{d} h(s)=[\lambda+\mu \ell(s)] h(s) \mathrm{d} s, \quad s \in[t, T], \\
x(t)=x>0, \quad h(t)=h>0 .
\end{array}\right.
$$

Remark 1. The above state equation admits a unique solution for any pair of measurable functions $(c(\cdot), \ell(\cdot))$. Denoting its value starting at time $t$ from $(x, h)$ and under the control $(c(\cdot), \ell(\cdot))$ by $x(t ; s, x, h, c(\cdot), l(\cdot))$ and $h(t ; s, x, h, c(\cdot), l(\cdot))$, respectively, we have 


$$
\begin{gathered}
x(s ; t, x, h, c, l)=x \mathrm{e}^{r(s-t)}+\int_{t}^{s}(\theta h(z) \ell(z)-c(z)) \mathrm{e}^{r(s-z)} \mathrm{d} z \\
h(s ; t, h, l)=h \exp \left\{\lambda(s-t)+\mu \int_{t}^{s} \ell(z) \mathrm{d} z\right\} .
\end{gathered}
$$

The objective functional becomes

$$
J(t, x, h ; c(\cdot), \ell(\cdot))=\int_{t}^{T} \mathrm{e}^{-\rho s} u(c(s), \ell(s)) \mathrm{d} s+\mathrm{e}^{-\rho T} b(h(T), x(T)),
$$

where $(c(\cdot), \ell(\cdot))$ belongs to the set of admissible controls $\mathcal{V}_{\mathrm{ad}}(t, x, h)$ defined as follows

$$
\begin{aligned}
\mathcal{V}_{\text {ad }}(t, x, h):= & \{(c(\cdot), \ell(\cdot)): \mathbb{T} \rightarrow \Gamma \mid(c(\cdot), \ell(\cdot)) \text { measurable, } \\
& x(s ; t, x, c(\cdot), \ell(\cdot))>0, s \in[t, T]\} .
\end{aligned}
$$

Definition 2. An admissible control $(\bar{c}(\cdot), \bar{\ell}(\cdot)) \in \mathcal{V}_{\text {ad }}(t, x, h)$ is called optimal for the initial condition $(t, x, h)$ if

$$
-\infty<J(t, x, h ; \bar{c}(\cdot), \bar{\ell}(\cdot))<+\infty
$$

and

$$
J(t, x, h ; c(\cdot), \ell(\cdot)) \leq J(t, x, h ; \bar{c}(\cdot), \bar{\ell}(\cdot)), \quad \forall(c(\cdot), \ell(\cdot)) \in \mathcal{V}_{\text {ad }}(t, x, h) .
$$

Set $\bar{x}(\cdot):=x(\cdot ; t, x, \bar{c}(\cdot), \bar{\ell}(\cdot))$ and $\bar{h}(\cdot):=x(\cdot ; t, h, \bar{c}(\cdot), \bar{\ell}(\cdot))$, the corresponding state trajectory $(\bar{x}(\cdot), \bar{h}(\cdot))$ is the optimal state trajectory. Furthermore, the pair $(\bar{x}(\cdot), \bar{h}(\cdot))$ along with the pair $(\bar{c}(\cdot), \bar{\ell}(\cdot))$ are known as an optimal state-control couple.

To solve our control problem we have to find the optimal couple $(\bar{c}(\cdot), \bar{\ell}(\cdot))$ and $(\bar{x}(\cdot), \bar{h}(\cdot))$, i.e., for any $(t, x, h) \in \mathbb{T}^{-} \times \Lambda$,

$$
\text { maximize } J(t, h, x ; c(\cdot), \ell(\cdot)) \text { over } \mathcal{V}_{\text {ad }}(t, x, h) .
$$

The above is a family of optimal control problems parameterized by $(t, x, h) \in \mathbb{T}^{-} \times \Lambda$ in which the original optimization problem with $t=0$, $x=x_{0}, h=h_{0}$ is embedded.

Remark 3. It is easy to show that the set of admissible strategies $\mathcal{V}_{\text {ad }}(t, x, h)$ is nonempty if we choose, e.g., any $(c, \ell) \in(0, r x] \times[0,1)$.

We aim to represent preferences that exhibit a constant rate of intertemporal substitution regarding a consumption-leisure bundle and exactly cancel out income and substitution effects. The resulting class of utility functions provides balanced growth, i.e., if labor grows at a constant rate then consumption growth at that same rate, but also a constant labor supply is awarded. It is well known that this class is given by CES (Constant Elasticity of Substitution) utility functions and there are many commonly used special cases. One is typically the logarithmic utility function. Therefore, following Romer (1996) we assume that the instantaneous utility function of the representative agent is represented by

$$
u(c(t), \ell(t)):=\alpha \ln c(t)+\beta \ln w(1-\ell(t)), \quad \alpha, \beta \in(0,1), \quad t \in \mathbb{T},
$$


while the bequest function corresponds to

$$
b(h(T), x(T)):=\frac{\alpha+\beta}{\rho} \ln (h(T)+x(T)), \quad \alpha, \beta \in(0,1), \quad \rho>0,
$$

i.e., the preference discounted value of the total residual amount of utility coming from consumption and leisure.

We just recall that the above utility function (7) is the limit as $\sigma \rightarrow 1$ of the following isoelastic utility function

$$
u(c, \ell)=\frac{\left[c^{\alpha}(w(1-\ell))^{\beta}\right]^{1-\sigma}-1}{1-\sigma} .
$$

where $\frac{1}{\sigma}$ is the intertemporal elasticity of substitution. The larger $\sigma$, the more consumption $c$ and income $w(1-\ell)$ are easily substitutable between time periods. A logarithmic utility function represents the preferences consistent with balanced growth and additively separable utility over consumption and leisure.

Moreover, $u_{c}>0, u_{c c}<0$, and $u_{\ell}<0, u_{\ell \ell}<0$, as expected.

\subsection{The Value Function and the Hamilton Jacobi Bellman Equation}

In order to proceed with the DP method, we have to consider the value function associated with the optimization problem and defined by

$$
\left\{\begin{array}{l}
V(t, h, x):=\sup _{(c \cdot(\cdot),(\cdot)) \in V_{\mathrm{ad}}(t, x, h)} J(t, h, x ; c(\cdot), \ell(\cdot)), \quad \forall(t, x, h) \in \mathbb{T}^{-} \times \Lambda, \\
V(T, h, x):=\mathrm{e}^{-\rho T} b(x, h), \quad \forall(x, h) \in \Lambda .
\end{array}\right.
$$

In the context of optimal control problems with finite horizon, the value function is formally associated with a nonlinear parabolic PDE with terminal boundary condition, which is the so-called Hamilton-Jacobi-Bellman (HJB hereafter) equation. In this case, the $\mathrm{HJB}$ equation reads as

$$
\left\{\begin{array}{l}
v_{t}(t, x, h)+\mathcal{H}\left(t, h, x, v_{x}, v_{h}\right)=0, \quad(t, x, h) \in \mathbb{T}^{-} \times \Lambda, \\
v(T, x, h)=\mathrm{e}^{-\rho T} b(h, x), \quad(x, h) \in \Lambda,
\end{array}\right.
$$

where

$$
\begin{aligned}
\mathcal{H}: & \mathbb{T} \times \Lambda \times \Lambda \rightarrow \mathbb{R}, \\
\left(t, x, h, p_{1}, p_{2}\right) & \mapsto \sup _{(c, \ell) \in \Gamma} \mathcal{H}_{c v}\left(t, h, x, v_{x}, v_{h} ; c, \ell\right)
\end{aligned}
$$

is the so-called Hamiltonian, whereas the function

$$
\begin{aligned}
\mathcal{H}_{c v}: & \mathbb{T} \times \Lambda \times \Lambda \times \Gamma \rightarrow \mathbb{R}, \\
\quad\left(t, x, h, p_{1}, p_{2} ; c, \ell\right) & \mapsto(\theta h \ell+r x-c) p_{1}+(\lambda+\mu \ell) h p_{2}+\mathrm{e}^{-\rho t} u(c, \ell)
\end{aligned}
$$

is the Hamiltonian current value.

It is easy to show that given $\left(t, x, h, p_{1}, p_{2}\right) \in \mathbb{T} \times \Lambda \times \Lambda$, the function $(c, \ell) \mapsto \mathcal{H}\left(t, x, h, p_{1}, p_{2} ; c, \ell\right)$ has a unique maximum point on $\Gamma$ given by 


$$
\begin{aligned}
& \left(\bar{c}\left(t, x, h, p_{1}, p_{2}\right), \bar{\ell}\left(t, x, h, p_{1}, p_{2}\right)\right) \\
& :=\arg \underset{(c, l) \in \Gamma}{\max _{c v}}\left(t, x, h, p_{1}, p_{2} ; c, \ell\right) \\
& =\left(\mathrm{e}^{-\rho t} \frac{\alpha}{p_{1}}, 1-\mathrm{e}^{-\rho t} \frac{\beta}{h\left(\theta p_{1}+\mu p_{2}\right)}\right) .
\end{aligned}
$$

Thus, the HJB equation is given by

$$
\begin{aligned}
& v_{t}(t, x, h)+(\theta h+r x) v_{x}(t, x, h)+(\lambda+\mu) h v_{h}(t, x, h) \\
& -\mathrm{e}^{-\rho t}\left[\alpha \ln v_{x}(t, x, h)+\beta \ln \left(\theta v_{x}(t, x, h)+\mu v_{h}(t, x, h)\right)\right] \\
& +\mathrm{e}^{-\rho t}[(\alpha+\beta) \rho+\gamma]=0, \quad(t, x, h) \in \mathbb{T}^{-} \times \Lambda,
\end{aligned}
$$

with the terminal condition

$$
v(T, x, h)=\frac{\alpha+\beta}{\rho} \mathrm{e}^{-\rho T} \ln (h+x), \quad(x, h) \in \Lambda,
$$

and where $\gamma:=\alpha(1-\ln \alpha)+\beta(1-\ln \beta \theta)$.

Definition 4 (Classical solution). A function $v$ is called a classical solution of (12a)-(12b) if:

(i) $v \in \mathcal{C}^{1,1}(\mathbb{T} \times \Lambda ; \mathbb{R})$;

(ii) $V$ satisfies pointwise in classical sense the partial differential Equation (12a) (the derivatives with respect to the time variable at $t=0$ and $t=T$ have to be intended as right and left derivatives respectively);

(iii) $v$ satisfies the boundary condition (12b).

\subsection{The Verification Theorem and the Optimal Feedback Policies}

We look for an explicit classical solution to the HJB Equation (12)) of the form

$$
v(t, x, h):=k \mathrm{e}^{-\rho t} \ln [a(t) x+b(t) h]
$$

for a suitable constant $k$ and functions $a, b \in \mathcal{C}^{1}(\mathbb{T} ; \mathbb{R})$. Substituting the above expression into the Equation (12), we see that we must have:

$$
k:=\frac{\alpha+\beta}{\rho},
$$

under the conditions

$$
\begin{gathered}
\rho \leq \mu \\
r \leq \lambda+\frac{\alpha+\beta}{\alpha \rho} \\
h_{0}>\frac{\mu}{\theta} x_{0} .
\end{gathered}
$$

The above inequalities are sufficient to grant that the no-bankruptcy constraint is fulfilled and the maximum point in the Hamiltonian $\mathcal{H}$ defined in (9) is such that $(\bar{c}, \bar{\ell}) \in \Gamma$. Otherwise, the above verification function $v$ could be the value function of a problem with a larger control set and whose optimal trajectory could not be admissible for our problem as well. 
We now give some lemmata that will be useful to prove the verification theorem, i.e., under the conditions (15) the function (13) with $k$ given by (14) is actually the value function of our problem and there exists an optimal state-control couple in the sense of Definition 2. As a byproduct, we will find a unique feedback optimal strategy and then, via the so-called closed loop equation, a unique optimal state-control couple.

Lemma 5. Let the conditions (15) be satisfied and $v$ be the function defined in (13) with $k$ given by (14), i.e.,

$$
v(t, x, h)=\frac{\alpha+\beta}{\rho} \mathrm{e}^{-\rho t} \ln [a(t) x+b(t) h], \quad \forall(t, x, h) \in \mathbb{T} \times \Lambda,
$$

where the pair of functions $a, b: \mathbb{T} \rightarrow \mathbb{R}$ is the unique solution to the following terminal value problem

$$
\left\{\begin{aligned}
\dot{a}(t)= & \left\{\frac{\alpha}{k} \ln a(t)+\frac{\beta}{k} \ln [\theta a(t)+\mu b(t)]+\rho \ln k+\frac{\gamma}{k}-r\right\} a(t), t \in[0, T), \\
\dot{b}(t)= & \left\{\frac{\alpha}{k} \ln a(t)+\frac{\beta}{k} \ln [\theta a(t)+\mu b(t)]+\rho \ln k+\frac{\gamma}{k}-\lambda-\mu\right\} b(t) \\
& -\theta a(t), t \in[0, T), \\
a(T)= & 1, \quad b(T)=1,
\end{aligned}\right.
$$

i.e., for any $t \in[0, T]$,

$$
\begin{aligned}
a(t)= & \exp \left\{\left[1-\frac{r}{\rho}+\ln \frac{\alpha+\beta}{\rho}-\frac{1}{\alpha+\beta}(\alpha \ln \alpha+\beta \ln [\beta \theta(\lambda+\mu-r)])\right]\left(\mathrm{e}^{-\rho(T-t)}-1\right)\right. \\
& \left.-\frac{\beta \rho}{\alpha+\beta} \mathrm{e}^{\rho t} \int_{t}^{T} \mathrm{e}^{-\rho s} \ln \left[\mu(\theta+\lambda+\mu-r) \mathrm{e}^{(\lambda+\mu-r)(T-s)}+\theta(\lambda-r)\right] \mathrm{d} s\right\}, \\
b(t)= & {\left[\frac{\theta}{\lambda+\mu-r}\left(\mathrm{e}^{(\lambda+\mu-r)(T-t)}-1\right)+\mathrm{e}^{(\lambda+\mu-r)(T-t)}\right] } \\
& \cdot \exp \left\{1-\frac{r}{\rho}+\ln \frac{\alpha+\beta}{\rho}-\frac{1}{\alpha+\beta}(\alpha \ln \alpha+\beta \ln [\beta \theta(\lambda+\mu-r)])\left(\mathrm{e}^{-\rho(T-t)}-1\right)\right. \\
& \left.-\frac{\beta \rho}{\alpha+\beta} \mathrm{e}^{\rho t} \int_{t}^{T} \mathrm{e}^{-\rho s} \ln \left[\mu(\theta+\lambda+\mu-r) \mathrm{e}^{(\lambda+\mu-r)(T-s)}+\theta(\lambda-r)\right] \mathrm{d} s\right\},
\end{aligned}
$$

if $\lambda+\mu-r \neq 0$, and

$$
\begin{aligned}
& \left\{\begin{aligned}
a(t)= & \exp \left\{\left[1-\frac{r}{\rho}+\ln \frac{\alpha+\beta}{\rho}-\frac{1}{\alpha+\beta}(\alpha \ln \alpha+\beta \ln \beta \theta)\right]\left(\mathrm{e}^{-\rho(T-t)}-1\right)\right. \\
& \left.-\frac{\beta \rho}{\alpha+\beta} \mathrm{e}^{\rho t} \int_{t}^{T} \mathrm{e}^{-\rho s} \ln [\mu+\theta+\mu \theta(T-s)] \mathrm{d} s\right\}, \\
b(t)= & {[1+\theta(T-t)] \exp \left\{1-\frac{r}{\rho}+\ln \frac{\alpha+\beta}{\rho}-\frac{1}{\alpha+\beta}(\alpha \ln \alpha+\beta \ln \beta \theta)\left(\mathrm{e}^{-\rho(T-t)}-1\right)\right.} \\
& \left.-\frac{\beta \rho}{\alpha+\beta} \mathrm{e}^{\rho t} \int_{t}^{T} \mathrm{e}^{-\rho s} \ln [\mu+\theta+\mu \theta(T-s)] \mathrm{d} s\right\},
\end{aligned}\right. \\
& \text { if } \lambda+\mu-r=0 .
\end{aligned}
$$


Then $v$ is a classical solution to the HJB Equation (12).

Proof. The claim follows a straightforward computation.

We may define feedback maps in the classical sense associated with the maximization of the Hamiltonian current value $\mathcal{H}_{c v}$ given by (10). To this end, consider the maximum point of $\mathcal{H}_{c v}$ when the gradient $\nabla v$ of the verification function (16), i.e.,

$$
\begin{aligned}
& \nabla v(t, x, h)=\left(v_{x}, v_{h}\right)(t, x, h) \\
& =\left(\mathrm{e}^{-\rho t} \frac{\alpha+\beta}{\rho} \frac{a(t)}{a(t) x+b(t) h}, \mathrm{e}^{-\rho t} \frac{\alpha+\beta}{\rho} \frac{b(t)}{a(t) x+b(t) h}\right),(t, x, h) \in \mathbb{T} \times \Lambda,
\end{aligned}
$$

is plugged in place of the formal argument $\left(p_{1}, p_{2}\right)$ in (11), that is

$$
\begin{aligned}
& (\bar{c}(t, x, h), \bar{\ell}(t, x, h)) \\
& =\left(\frac{\alpha \rho}{\alpha+\beta}\left[x+\frac{b(t)}{a(t)} h\right], 1-\frac{\beta \rho}{\alpha+\beta} \frac{a(t) x+b(t) h}{[\theta a(t)+\mu b(t)] h}\right), \quad(t, x, h) \in \mathbb{T} \times \Lambda .
\end{aligned}
$$

Lemma 6. Assume that condition (15b) holds, i.e., $r \leq \lambda+\frac{\alpha+\beta}{\alpha \rho}$, and let $\tau \in \mathbb{T}^{-}$such that $h(\tau)>\frac{\mu}{\theta} x(\tau)>0$. Then, there exists $0<\delta \leq T-\tau$ such that

$$
\frac{\mathrm{d}}{\mathrm{d} t} h(t)-\frac{\mu}{\theta} \frac{\mathrm{d}}{\mathrm{d} t} x(t)>0, \quad \forall t \in[\tau, \tau+\delta] \subseteq \mathbb{T}
$$

Furthermore

$$
\frac{\mu}{\theta} x(t)>0, \quad \forall t \in[\tau, \tau+\delta] \subseteq \mathbb{T} .
$$

Proof. See Appendix.

Lemma 7. Assume that conditions (15b)-(15c) hold, i.e., $r \leq \lambda+\frac{\alpha+\beta}{\alpha \rho}$ and $h_{0}>\frac{\mu}{\theta} x_{0}$. Then $h(t)>\frac{\mu}{\theta} x(t)>0$, for any $t \in \mathbb{T}$.

Proof. See Appendix.

As a straightforward consequence of the previous Lemma 7, we have that for any $(t, x, h) \in \mathbb{T} \times \Lambda$ the gradient $\nabla v(t, x, h)$ specified in (18) is component-wise strictly positive and allows us to show that the feedback map $(\bar{c}(t, x, h), \bar{\ell}(t, x, h))$ stated in (19) has a key feature. This is done in the following proposition.

Proposition 8. Let conditions (15) hold. Then $(\bar{c}(t, x, h), \bar{\ell}(t, x, h))$ pointed out in (19), for any initial data $(t, x, h) \in \mathbb{T} \times \Lambda$, is such that

$$
\bar{c}(t, x, h)>0 \wedge 0 \leq \bar{\ell}(t, x, h)<1 .
$$

Proof. See Appendix.

The closed loop equation, obtained by replacing $c(\cdot), l(\cdot)$ with $\bar{c}(\cdot, x, h)$, $\bar{\ell}(\cdot, x, h)$ stated in (19), respectively, into dynamics (1)-(3), for every 
$(x, h) \in \Lambda$, read as

$$
\left\{\begin{array}{l}
\mathrm{d} x(s)=[w(s) \bar{\ell}(s, x(s), h(s))-\bar{c}(s, x(s), h(s))+r x(s)] \mathrm{d} s, \quad s \in[t, T], \\
\mathrm{d} h(s)=(\lambda+\mu \bar{\ell}(s, x(s), h(s))) h(s) \mathrm{d} s, \quad s \in[t, T], \\
x(t)=x, \quad h(t)=h,
\end{array}\right.
$$

namely,

$$
\begin{aligned}
\mathrm{d} x(s)= & \left\{\left[r-\frac{\alpha \rho}{\alpha+\beta}-\frac{1}{\alpha+\beta} \frac{\beta \theta a(s)}{\mu b(s)}\right] x(s)\right. \\
& \left.+\left[\theta-\frac{1}{\alpha+\beta}\left(\alpha+\frac{\beta \theta a(s)}{\theta a(s)+\mu b(s)}\right)\right] h(s)\right\} \mathrm{d} s, \quad s \in[t, T], \\
\mathrm{d} h(s)=\{ & -\frac{1}{\alpha+\beta} \frac{\beta \mu a(s)}{\theta a(s)+\mu b(s)} x(s) \\
& \left.+\left[\lambda+\mu-\frac{1}{\alpha+\beta} \frac{\beta \mu b(s)}{\theta a(s)+\mu b(s)}\right] h(s)\right\} \mathrm{d} s, \quad s \in[t, T], \\
x(t)=x, & h(t)=h .
\end{aligned}
$$

The solution of the above system of differential equations has a desirable feature that is stated in the following lemma.

Lemma 9 (Closed loop equation). Let conditions (15) hold. For every $(t, x, h) \in \mathbb{T} \times \Lambda$, there exists a unique pair of function $(\bar{x}(\cdot), \bar{h}(\cdot)):=(\bar{x}(\cdot ; t, x, h), \bar{h}(\cdot ; t, x, h)) \in \mathcal{C}^{1}(\mathbb{T} ; \Lambda)$ solution to closed loop Equation (22).

Proof. See Appendix.

Solving an optimal control problem requires finding an optimal control and the corresponding state trajectory. The main motivation of introducing dynamic programming is that one might able to construct an optimal feedback control via the value function.

Definition 10. A pair of measurable function $(c, \ell): \mathbb{T} \times \Lambda \rightarrow \Gamma$ is called an admissible feedback control if for any $(t, x, h) \in \mathbb{T}^{-} \times \Lambda$, there is a solution to the following closed loop equation

$$
\left\{\begin{array}{l}
\mathrm{d} x(s)=[w(s) \ell(t, x(s), h(s))-c(t, x(s), h(s))+r x(s)] \mathrm{d} s, \quad s \in[t, T] \\
\mathrm{d} h(s)=[\lambda+\mu \ell(t, x(s), h(s))] h(s) \mathrm{d} s, \quad s \in[t, T] \\
x(t)=x>0, \quad h(t)=h>0 .
\end{array}\right.
$$

An admissible feedback control $\left(c^{\star}, \ell^{\star}\right)$ is said to be optimal if for each $(t, x, h) \in \mathbb{T}^{-} \times \Lambda, \quad\left(x^{\star}(\cdot), h^{\star}(\cdot), c^{\star}(\cdot), \ell^{\star}(\cdot)\right):=$ $\left(x^{\star}(\cdot ; x, h), h^{\star}(\cdot ; x, h), c^{\star}\left(; x^{\star}(; x, h), h^{\star}(; x, h)\right), \ell^{\star}\left(; x^{\star}(\cdot ; x, h), h^{\star}(\cdot ; x, h)\right)\right)$ is optimal for problem (6), where $\left(x^{\star}(\cdot), h^{\star}(\cdot)\right):=\left(x^{\star}(\cdot ; x, h), h^{\star}(\cdot ; x, h)\right)$ is the solution to (23) corresponding to $\left(c^{\star}, \ell^{\star}\right)$.

Before to give the main result, state a fundamental identity in the next lemma. 
Lemma 11 (Fundamental identity). Let conditions (15) be satisfied and $v$ be the function defined in (16). Then, for every initial data $(t, x, h) \in \mathbb{T} \times \Lambda$ and pair $(c(\cdot), \ell(\cdot)) \in \mathcal{V}_{\text {ad }}(t, x, h)$ the following fundamental identity holds

$$
\begin{aligned}
& v(t, x, h)=J(t, x, h ; c(\cdot), \ell(\cdot)) \\
& +\int_{t}^{T}\left[\mathcal{H}\left(s, x(s), h(s), v_{x}(s, x(s), h(s)), v_{h}(s, x(s), h(s))\right)\right. \\
& \left.-\mathcal{H}_{c v}\left(s, x(s), h(s), v_{x}(s, x(s), h(s)), v_{h}(s, x(s), h(s)) ; c(\cdot), \ell(\cdot)\right)\right] \mathrm{d} s
\end{aligned}
$$

where $(x(\cdot), h(\cdot)):=(x(\cdot ; t, x, h, c(\cdot)), h(\cdot ; t, x, h, c(\cdot), \ell(\cdot)))$, and the functions $\mathcal{H}, \mathcal{H}_{c v}$ are defined in (9), (10), respectively.

Proof. See Appendix.

At this point, our goal is to formulate the main result of this section, i.e., a verification theorem proving that the function $v$ defined in (16) is actually the value function $V$ defined in (8) and construct an optimal feedback control via the value function.

Theorem 12 (Verification theorem). Let conditions (15) be satisfied, and $v$ be the function defined in (16). Then, for any $(t, x, h) \in \mathbb{T} \times \Lambda$,

$$
V(t, x, h)=v(t, x, h) \text {. }
$$

Furthermore, the strategy starting at $(t, x, h) \in \mathbb{T}^{-} \times \Lambda$ and associated to the maximization of the Hamiltonian current value $\mathcal{H}_{c v}$ is the unique optimal feedback control in the sense of Definition 10, namely,

$$
\left(c^{\star}(s), \ell^{\star}(s)\right):=\left(\frac{\alpha}{k}\left[x^{\star}(s)+\frac{b(s)}{a(s)} h^{\star}(s)\right], 1-\frac{\beta}{k} \frac{\frac{x^{\star}(s)}{h^{\star}(s)}+\frac{b(s)}{a(s)}}{\theta+\mu \frac{b(s)}{a(s)}}\right), s \in[t, T] .
$$

Proof. See Appendix.

\section{Economic Implications of the Optimal Strategies}

From the previous section, we know that the unique optimal strategy starting at $(t, x, h) \in \mathbb{T}^{-} \times \Lambda$ given in feedback form is given by (25). The optimal consumption choice $c^{\star}(\cdot)$ has the following stylized properties:

(i) according to the Permanent Income Hypothesis by Friedman (1957), the representative agent decides her optimal consumption strategy taking into account her equivalent total wealth level, i.e., $a(\cdot) x^{\star}(\cdot)+b(\cdot) h^{\star}(\cdot)$;

(ii) the optimal consumption strategy is increasing with the equivalent total wealth;

while the optimal labor choice $\ell^{\star}(\cdot)$ has the following stylized features:

(iii) the optimal labor policy decreases as the total equivalent wealth grows;

(iv) the optimal labor strategy increases as $\frac{x^{\star}(\cdot)}{h^{\star}(\cdot)}$, i.e., the ratio between liquid wealth and human capital, decreases: at fixed liquid wealth level, the higher the 
human capital the higher the labor level and vice-versa.

\subsection{Parameters Significance}

In order to explore the peculiar implications of the above findings for economic analysis, it is useful to recall the basic significance of the parameters in the model:

$r$ : rate of return of financial wealth;

$\lambda+\frac{1}{\rho} \frac{\alpha+\beta}{\alpha}$ : maximum rate of return of financial wealth preventing the agent is willing to work more than the maximum allowed intensity and consume less than zero;

$\lambda:$ rate of growth of human capital when the agent exerts the minimum work intensity $l=0$;

$\mu:$ is the marginal increase in the rate of return of human capital with respect to labor supply, i.e., it represents the intensity of learning per unit of labor supply;

$\lambda+\mu$ : rate of growth of human capital when the agent exerts the maximum work intensity $l=1$;

$\theta$ : the liquid value of work per unit of human capital.

\subsection{Optimal Control Policies When the Transversality Condition Holds}

If the transversality condition

$$
r-(\lambda+\mu)>0
$$

holds, then the Gordon Growth Formula can be applied to define a liquid price of the stock of human capital. In fact, for $T \rightarrow+\infty$ the marginal rate of substitution between financial and human capital reads as

$$
\lim _{T \rightarrow+\infty} \frac{\partial V / \partial h}{\partial V / \partial x}=\frac{\theta}{r-\lambda-\mu},
$$

and thus the liquidation value of a stock of human capital $h^{\star}$ is given by

$$
\frac{\theta}{r-\lambda-\mu} h^{\star}
$$

The optimal consumption policy follows the classical Fri Permanent Income Hypothesis, i.e. the agent consumes a fraction of total equivalent wealth

$$
c^{\star}(\cdot)=\rho \frac{\alpha}{\alpha+\beta}\left(x^{\star}(\cdot)+\frac{\theta}{r-\lambda-\mu} h^{\star}(\cdot)\right) .
$$

In order to fully assess the role of the parameter $\mu$ it is necessary to consider its multiple roles. First, since

$$
\theta+\mu \frac{b(\cdot)}{a(\cdot)} \stackrel{T \rightarrow+\infty}{\longrightarrow} \theta\left(1+\frac{\mu}{r-\lambda-\mu}\right),
$$

we observe that it determines the effective marginal liquid value per unit of hu- 
man capital $h$ including driven by the learning-by-doing effect. Thus, raising $\mu$, also the effective present liquid dividend of the human capital will rise. Correspondingly, the optimal labor supply is determined by

$$
\begin{array}{r}
l^{\star}(\cdot)=1-\left(1-\frac{\alpha}{\alpha+\beta}\right) \frac{\rho}{\mu} \frac{\frac{x^{\star}(\cdot)}{\theta h^{\star}(\cdot)}+\frac{1}{\mu} \frac{r-\lambda}{\mu}-1}{1+\frac{1}{\frac{r-\lambda}{\mu}-1}} \\
l^{\star}(\cdot)=1-\frac{\beta}{\alpha+\beta} \frac{\rho}{\mu} \frac{\frac{x^{\star}(\cdot)}{\mu h^{\star}(\cdot)}+\frac{1}{\frac{r-\lambda}{\mu}-1}}{1+\frac{1}{\frac{r-\lambda}{\mu}-1}}
\end{array}
$$

Recalling (2) and (15c), i.e.,

$$
w(\cdot)=\theta h(\cdot) \quad \text { and } \quad h_{0}>\frac{\mu}{\theta} x_{0},
$$

we notice that a sufficient condition to have a well defined $l^{\star}>0$ is

$$
x_{0} \leq \frac{\theta h_{0}}{\mu}=\frac{w_{0}}{\mu},
$$

where $w_{0}=: w(0)$. If this requirement is satisfied at time 0 , then the same condition will be verified for any time $t \in \mathbb{T}$. The above conditions are sufficient to guarantee that there exists an effective tradeoff (an internal solution) between the supply of labor, the level of leisure achievable by the household and the level of consumption that can be obtained out of liquid savings.

Consider

$$
\frac{w_{0}}{\mu},
$$

as the liquid present value of the potential human capital at initial time 0 that is achievable through learning-by-doing by a worker with an initial wage level $w_{0}=\theta h_{0}$ equivalent to an initial level of human capital $h_{0}$. Observe that the parameter $\mu$ determines the rate of growth of human capital driven by learning-by-doing. As in the case of interest rates, its increase determines also a reduction of the present value of human capital. In other words, the higher the level of $\mu$ the higher the value of the working experience.

The condition (15a), i.e.,

$$
\rho \leq \mu
$$

states that the rate of preference discount is not bigger than $\mu$, the marginal increase in the rate of return of human capital per unit of labor supply. This condition requires that the agent is sufficiently patient in order to appreciate the 
value of working experience.

Observe that the higher the level of financial wealth $x$ the higher the level of leisure for a fixed level of human capital $h$.

The optimal level of labor supply will be increasing in the discount rate of preference $\rho$, increasing (decreasing) in the relative elasticity $\frac{\alpha}{\alpha+\beta}\left(\frac{\beta}{\alpha+\beta}\right)$ of utility with respect to consumption (leisure).

The transversality condition can also be rewritten as

$$
\frac{r-\lambda}{\mu}>1 \text {. }
$$

It states that the rate of return on financial capital net of the rate of human capital determined by innate capabilities must be larger than $\mu$, i.e., the marginal increase in the rate of return of human capital per unit of labor supply. It is interesting to observe that the role played by learning-by-doing and innate abilities in this analysis is opposite.

\subsection{Human Capital Accumulation in a Low Interest Rates Regime}

It is interesting to observe that the model will produce an optimal solution also when the transversality condition does not apply.

In this situation the optimal policy is no more time homogeneous. In general it will depend on the distance from the final liquidation time. In fact time $T$ determines the future time where the marginal rate of substitution between financial capital and human capital equals 1 by construction.

In practice, lack of stationarity implies that both the total equivalent level of wealth

$$
x^{\star}(t)+\frac{b(t)}{a(t)} h^{\star}(t), \quad t \in \mathbb{T},
$$

and the effective financial liquidation value of human capital per unit of labor supply

$$
\theta+\mu \frac{b(t)}{a(t)}, \quad t \in \mathbb{T},
$$

will be time-dependent and nonstationary. We can rationalize this situation as a condition of a household that is accumulating human capital and financial wealth during the lifecycle with a predetermined target that will change its ability to liquidate human capital. Consider for example an entrepreneur that is willing to maximize the value of the enterprise before a predetermined initial public offering when she will liquidate her share of stock in a startup or a Ph.D. student that is willing to maximize the rate of accumulation of human capital before a predetermined date, for example before terminating the $\mathrm{Ph} . \mathrm{D}$. and applying for positions in the academic job market. In these cases, the role of the learning-by-doing is also relevant in relation to the time missing before the li- 
quidation event.

Remarkably, the same type of analysis would apply in a situation where financial activities have no longer an expected rate of return $r<\lambda+\mu$. This may be, e.g., the situation of a market where financial activities have prices so high that the rate of return is certainly lower than the rate f growth of human capital under maximum labor supply. Then the critical time will correspond to the liquidation time, where the accumulated human capital becomes equivalent to liquid wealth. Correspondingly, all the optimal policies will be parameterized with the time to the critical liquidation time.

The above analysis highlights the inability to exploit learning-by-doing wage growth potential by short-term temporary workers that switch frequently between jobs requiring different skills. In practice, the non-linearity implicitly in the human capital accumulation amplifies the reduction in lifetime earnings and in the resulting welfare implications. From a general equilibrium point of view it would be interesting to explore the optimal tradeoff between the production flexibility implied by a mobile workforce and the implicit relevant costs that labor flexibility has in terms of generating human capital obsolescence and the social problems that may derive from it.

Notice also that the higher the liquid-to-human wealth ratio $\frac{x^{\star}}{h^{\star}}$, the lower the optimal level of effort that is required to maximize welfare, this relationship is stronger the lower the liquid value of human capital as determined by the ratio $\frac{b}{a}$. This implies that different levels of liquid compensation of professional (human capital) skills may drive up or down the working incentives, the productivity and in ultimate analysis it may heavily affect lifetime earning distribution and social mobility.

An endogenous source of discontinuous careers is also a faster rate of technical change, leading to anticipated obsolescence of workers' skills and the need to move more frequently from contracting to expanding industry sectors.

\section{Conclusion, Limitations, and Further Research}

The above findings highlight the relevant role that non-linearity of the human capital dynamics created by the learning-by-doing action on wage growth has crucial implications on the individual lifetime earnings and on wealth growth. There are two equally important directions to improve the above analysis by making it suitable to match with panel data on labor and census. The first one is to introduce exogenous uncertainty in the accumulation of liquid and human wealth assuming that parameters are known and depend on the structural characteristics of the economy and on innate human characteristics.

Last but not least, the introduction of additional impulse control to model the optimal job ladder policy and the risk and opportunities deriving from job loss and by different levels of job finding rates in order would provide an interesting 
framework to run a comparative analysis of the welfare costs and on inequality. Our model does not allow the derivation of robust policy implications. The latter step goes beyond the scope of this theoretical paper. Yet, it offers some clues on one side, on the role of education in shaping learning opportunities and workers' careers and, on the other side, on the impact that, discontinuous careers, due to labour market flexibility and/or a faster rate of skills obsolescence, may have on workers' wellbeing in a lifecycle perspective. An alternative way of assessing this impact is to consider explicitly the increasing cost for society of training due to faster technical change.

\section{Conflicts of Interest}

The authors declare no conflicts of interest regarding the publication of this paper.

\section{References}

Acemoglu, D., \& Autor, D. H. (2011). Chap. B. Skills, Tasks and Technologies: Implications for Employment and Earnings. In D. Card, \& O. Ashenfelter (Eds.), Handbook of Labor Economics (Vol. 4, pp. 1043-1171). North Holland. https://doi.org/10.1016/S0169-7218(11)02410-5

Autor, D. H., \& Dorn, D. (2013). The Growth of Low-Skill Service Jobs and the Polarization of the US Labor Market. The American Economic Review, 103, 1553-1597. https://doi.org/10.1257/aer.103.5.1553

Autor, D. H., Levy, F., \& Murnane, R. J. (2003). The Skill Content of Recent Technological Change: An Empirical Exploration. The Quarterly Journal of Economics, 118, 1279-1333. https://doi.org/10.1162/003355303322552801

Bardi, M., \& Capuzzo-Dolcetta, I. (1997). Optimal Control and Viscosity Solutions of Hamilton-Jacobi-Bellman Equations. Birkhauser. https://doi.org/10.1007/978-0-8176-4755-1

Becker, G. S. (1964). A Theoretical and Empirical Analysis, with Special Reference to Education. Columbia University Press for the National Bureau of Economic Research.

Blandin, A., \& Peterman, W. B. (2019). Taxing Capital? The Importance of How Human Capital Is Accumulated. European Economic Review, 119, 482-508.

https://doi.org/10.1016/j.euroecorev.2019.08.007

Brunello, G., Garibaldi, P., \& Wasmer, E. (2007). Education and Training in Europe. Oxford University Press. https://doi.org/10.1093/acprof:oso/9780199210978.001.0001

Chang, Y., Gomes, J. F., \& Schorfheide, F. (2002). Learning-by-Doing as a Propagation Mechanism. American Economic Review, 92, 1498-1520. https://doi.org/10.1257/000282802762024601

Chuna, F., \& Heckman, J. J. (2007). The Technology of Skill Formation. The American Economic Review, 97, 31-47. https://doi.org/10.1257/aer.97.2.31

Cossa, R., Heckman, J. J., \& Lochner, L. (2002). Wage Subsidies and Skill Formation: A Study of the Earned Income Tax Credit. NBER Working Paper No. 9083. http://www.nber.org/papers/w9083

D’Orlando, F., Ferrante, F., \& Oliverio, A. (2021). Economic Change and Well Being. In The True Costs of Creative Destruction and Globalization (p. 144). Routledge. https://doi.org/10.4324/9781003018230 
Friedman, M. (1957). A Theory of the Consumpion Function. Princeton University Press.

Hanushek, E. A., Schwerdt, G., Woessmann, L., \& Zhang, L. (2017). General Education, Vocational Education, and Labor-Market Outcomes over the Life-Cycle. The Journal of Human Resources, 52, 49-88. https://doi.org/10.3368/jhr.52.1.0415-7074R

Heckman, J. J., Stixrud, J., \& Urzua, S. (2006). The Effects of Cognitive and Noncognitive Abilities on Labor Market Outcomes and Social Behaviour. Journal of Labor Economics, 24, 411-482. https://doi.org/10.1086/504455

Imai, S., \& Keane, M. P. (2004). Intertemporal Labor Supply and Human Capital Accumulation. International Economic Review, 45, 601-641. https://doi.org/10.1111/j.1468-2354.2004.00138.x

Karahan, F., Ozkan, S., \& Song, J. (2019). Anatomy of Lifetime Earnings Inequality: Heterogeneity in Job Ladder Risk vs. Human Capital. Staff Report No. 908, Federal Reserve Bank of New York. https://doi.org/10.2139/ssrn.3511647

Lucas, R. E. J. (1988). On the Mechanism of Economic Development. Journal of Monetary Economics, 22, 3-42. https://doi.org/10.1016/0304-3932(88)90168-7

MaCurdy, T. E. (1999). An Essay on the Life Cicle: Characterizing Intertemporal Behavior with Uncertainty, Human Capital, Taxes, Durables, Imperfect Capital Markets, and Non-Separable Preferences. Research in Economics, 53, 5-46. https://doi.org/10.1006/reec.1998.0185

OECD (2013). OECD Skills Outlook 2013: First Results from the Survey of Adult Skills. OECD Publishing.

OECD (2018). Good Jobs for All in the Changing World of Work. The OECD Job Strategy. OECD Publishing. https://doi.org/10.1787/9789264308817-en

Roese, N. J., \& Summerville, A. (2005). What We Regret Most... and Why. Personality and Social Psychology Bulletin, 31, 1273-1285.

https://doi.org/10.1177/0146167205274693

Romer, P. M. (1990). Endogenous Technological Change. Journal of Political Economy, 98, S71-S102. https://doi.org/10.1086/261725

Romer, P. M. (1996). Advanced Macroeconomics. McGraw Hill.

Schultz, T. W. (1963). The Economic Value of the Education. Columbia Univerity Press.

Shaw, K. L. (1989). Life-Cycle Labor Supply with Human Capital Accumulation. International Economic Review, 30, 431-456. https://doi.org/10.2307/2526656

Uzawa, H. (1965). Optimum Technical Change in a Aggregative Model of Economic Growth. International Economic Review, 6, 18-31. https://doi.org/10.2307/2525621

Yong, J., \& Zhou, X. Y. (1999). Stochastic Controls: Hamiltonian Systems and HJB Equations. Springer Verlag. https://doi.org/10.1007/978-1-4612-1466-3 


\section{Appendix: Technical Proofs}

Here we provide the technical proofs.

\section{Proof of Lemma 6}

Setting

$$
y(t):=\frac{\mu}{\theta} x(t), \quad \forall t \in \mathbb{T}, \quad \text { and } \quad y_{0}:=\frac{\mu}{\theta} x_{0},
$$

the system of ordinary differential Equation (22) reads as

$$
\left\{\begin{aligned}
\frac{\mathrm{d} y(t)}{\mathrm{d} t}= & {\left[r-\frac{\rho}{\alpha+\beta}\left(\alpha+\frac{\beta \theta a(t)}{\theta a(t)+\mu b(t)}\right)\right] y(t) } \\
& +\mu\left[1-\frac{\rho b(t)}{\alpha+\beta}\left(\frac{\alpha}{\theta a(t)}+\frac{\beta}{\theta a(t)+\mu b(t)}\right)\right] h(t), \quad t \in \mathbb{T}, \\
\frac{\mathrm{d} h(t)}{\mathrm{d} t}= & -\frac{\beta \theta \rho a(t)}{(\alpha+\beta)(\theta a(t)+\mu b(t))} y(t) \\
& +\left[\lambda+\mu-\frac{\beta \mu \rho b(t)}{(\alpha+\beta)(\theta a(t)+\mu b(t))}\right] h(t), \quad t \in \mathbb{T}, \\
y(0)= & y_{0}>0, \quad h(0)=h_{0}>0 .
\end{aligned}\right.
$$

Notice that the above system admits a unique and continuous solution.

Let $\tau \in \mathbb{T}^{-}$such that $h(\tau)>y(\tau)>0$, and consider the following ordinary differential equation

$$
\left\{\begin{aligned}
\frac{\mathrm{d} \tilde{y}}{\mathrm{~d} t}(t)= & \left\{r-\frac{\rho}{\alpha+\beta}\left(\alpha+\frac{\beta \theta a(t)}{\theta a(t)+\mu b(t)}\right)\right. \\
& \left.+\mu\left[1-\frac{\rho b(t)}{\alpha+\beta}\left(\frac{\alpha}{\theta a(t)}+\frac{\beta}{\theta a(t)+\mu b(t)}\right)\right]\right\} \tilde{y}(t), \quad t \in[\tau, T], \\
\tilde{y}(\tau)= & y(\tau)>0,
\end{aligned}\right.
$$

whose unique solution is continuous and clearly $\tilde{y}(t)>0$, for any $t \in[\tau, T]$, since it is homogeneous and $\tilde{y}(0)>0$. Moreover, there exists $0<\delta \leq T-\tau$ such that, for any $t \in[\tau, \tau+\delta]$, we have

$$
\begin{aligned}
\frac{\mathrm{d}}{\mathrm{d} t} y(t) & =\left[r-\frac{\rho}{\alpha+\beta}\left(\alpha+\frac{\beta \theta a(t)}{\theta a(t)+\mu b(t)}\right)\right] y(t) \\
& +\left[\mu-\frac{\alpha \mu \rho}{\theta(\alpha+\beta)} \frac{b(t)}{a(t)}-\frac{\beta \mu \rho}{\alpha+\beta} \frac{b(t)}{\theta a(t)+\mu b(t)}\right] h(t) \\
> & {\left[r-\frac{\alpha \rho}{\alpha+\beta}-\frac{\beta \theta \rho}{\alpha+\beta} \frac{a(t)}{\theta a(t)+\mu b(t)}\right.} \\
& \left.+\mu-\frac{\alpha \mu \rho}{\theta(\alpha+\beta)} \frac{b(t)}{a(t)}-\frac{\beta \mu \rho}{\alpha+\beta} \frac{b(t)}{\theta a(t)+\mu b(t)}\right] \tilde{y}(t)=\frac{\mathrm{d}}{\mathrm{d} t} \tilde{y}(t),
\end{aligned}
$$

and as a byproduct we obtain $y(t)>0$, for any $t \in[\tau, \tau+\delta]$, since in the time 
interval under consideration the inequalities $y(t)>\tilde{y}(t)>0$ also hold.

In light of the above findings, notice that if condition (15b) is satisfied, we obtain

$$
\frac{\mathrm{d}}{\mathrm{d} t} h(t)-\frac{\mathrm{d}}{\mathrm{d} t} y(t)>0, \quad \forall t \in[\tau, \tau+\delta] .
$$

In fact, taking into consideration (26), for any $t \in[\tau, \tau+\delta]$, we have

$$
\begin{aligned}
\frac{\mathrm{d}}{\mathrm{d} t} h(t)-\frac{\mathrm{d}}{\mathrm{d} t} y(t)= & -\frac{\beta \theta \rho a(t)}{(\alpha+\beta)(\theta a(t)+\mu b(t))} y(t) \\
& +\left[\lambda+\mu-\frac{\beta \mu \rho b(t)}{(\alpha+\beta)(\theta a(t)+\mu b(t))}\right] h(t) \\
& -\left[r-\frac{\rho}{\alpha+\beta}\left(\alpha+\frac{\beta \theta a(t)}{\theta a(t)+\mu b(t)}\right)\right] y(t) \\
& -\mu\left[1-\frac{\rho b(t)}{\alpha+\beta}\left(\frac{\alpha}{\theta a(t)}+\frac{\beta}{\theta a(t)+\mu b(t)}\right)\right] h(t) \\
= & {\left[\lambda+\frac{\alpha \mu \rho}{\theta(\alpha+\beta)} \frac{b(t)}{a(t)}\right] h(t)+\left(\frac{\alpha \rho}{\alpha+\beta}-r\right) y(t) } \\
> & {\left[\lambda+\frac{\alpha \mu \rho}{\theta(\alpha+\beta)} \frac{b(t)}{a(t)}+\frac{\alpha \rho}{\alpha+\beta}-r\right] y(t)>0 . }
\end{aligned}
$$

Notice that if condition (15b) is fulfilled, the above inequality also applies at $t=T$ recalling that $a(T)=b(T)=1$.

\section{Proof of Lemma 7}

We proceed by contradiction showing that it is not possible to reach a point $t \in \mathbb{T}$ such that $h(t) \leq \frac{\mu}{\theta} x(t)$ unless having $h_{0} \leq \frac{\mu}{\theta} x_{0}$.

As in the previous lemma, for convenience we set $y(t):=\frac{\mu}{\theta} x(t)$ for any $t \in \mathbb{T}$. Since the solution to the system of ordinary differential Equation (26) is continuous, in order to have $h(t) \leq y(t)$, for some $t \in \mathbb{T}$, along with $h_{0}>y_{0}$, it is necessary that there exists a point $\tilde{t} \in \mathbb{T}^{-}$and $\delta>0$ such that $\left[\tilde{t}-\frac{\delta}{2}, \tilde{t}+\frac{\delta}{2}\right] \subseteq \mathbb{T}$ and $h\left(\tilde{t}-\frac{\delta}{2}\right)>y\left(\tilde{t}-\frac{\delta}{2}\right)$ while

$$
h\left(\tilde{t}+\frac{\delta}{2}\right) \leq y\left(\tilde{t}+\frac{\delta}{2}\right) .
$$

On the other hand, setting $\tau:=\tilde{t}-\frac{\delta}{2}$ we have $h(\tau)>y(\tau)$ and consequently Lemma 6 grants $\frac{\mathrm{d}}{\mathrm{d} t} h(\tau)-\frac{\mathrm{d}}{\mathrm{d} t} y(\tau)>0$. The latter inequality implies that there exists $0<\delta \leq T-\tau$ such that $h(t)>y(t)$ for any $t \in[\tau, \tau+\delta]$. In fact, by definition of derivative we have 


$$
\lim _{t \rightarrow \tau^{+}}\left(\frac{h(t)-h(\tau)}{t-\tau}-\frac{y(t)-y(\tau)}{t-\tau}\right)=\frac{\mathrm{d}}{\mathrm{d} t} h(\tau)-\frac{\mathrm{d}}{\mathrm{d} t} y(\tau)>0,
$$

and by the theorem of the permanence of sign there exist $(\tau, \tau+\delta] \subset \mathbb{T}$ such that for any $t \in(\tau, \tau+\delta]$ we obtain

$$
\frac{h(t)-h(\tau)}{t-\tau}-\frac{y(t)-y(\tau)}{t-\tau}>0 .
$$

Now, setting $t:=\tau+\delta$, since the denominator of the above left hand side is positive we get

$$
h(\tau+\delta)-h(\tau)-(y(\tau+\delta)-y(\tau))>0
$$

i.e.,

$$
h(\tau+\delta)>y(\tau+\delta)+\underbrace{h(\tau)-y(\tau)}_{>0} .
$$

Hence

$$
h(\tau+\delta)>y(\tau+\delta)
$$

i.e.,

$$
h\left(\tilde{t}+\frac{\delta}{2}\right)>y\left(\tilde{t}+\frac{\delta}{2}\right),
$$

which is clearly in contradiction with (27). Therefore, we must conclude that such a crossing point $\tilde{t}$ cannot exist. Moreover, for any $t \in[\tau, \tau+\delta]$, since $y(t)>0$ so $h(t)>0$ and the claim is proved.

\section{Proof of Proposition 8}

Recall (19) for convenience

$$
\left\{\begin{array}{l}
c^{\star}(t, x, h)=\frac{\alpha \rho}{\alpha+\beta}\left[x+\frac{b(t)}{a(t)} h\right], \quad(t, x, h) \in \mathbb{T} \times \Lambda, \\
\ell^{\star}(t, x, h)=1-\frac{\beta \rho}{\alpha+\beta} \frac{a(t) x+b(t) h}{[\theta a(t)+\mu b(t)] h}, \quad(t, x, h) \in \mathbb{T} \times \Lambda .
\end{array}\right.
$$

Since Lemma 7 holds and $a(t), b(t)>0$, for any $t \in \mathbb{T}$, then clearly $c^{\star}(t, x, h)$ is positive while $\ell^{\star}(t, x, h)$ is less than 1 , for any $(t, x, h) \in \mathbb{T} \times \Lambda$.

It remains to prove that $\ell^{\star}(t, x, h) \geq 0$, for any $(t, x, h) \in \mathbb{T} \times \Lambda$. The inequality holds as soon as

$$
\frac{\beta \rho}{\alpha+\beta} \frac{a(t) x+b(t) h}{[\theta a(t)+\mu b(t)] h} \leq 1,
$$

i.e.,

$$
\frac{\beta \rho}{(\alpha+\beta) \mu} \frac{a(t) \frac{X}{h}+b(t)}{\frac{\theta}{\mu} a(t)+b(t)} \leq 1,
$$


which is granted thanks to Lemma (7) and as soon as $\rho \leq \mu$, namely, condition (15a) is satisfied.

\section{Proof of Lemma 9}

For any initial data $(t, x, h) \in \mathbb{T} \times \Lambda$, the map $\bar{c}(t, x, h)$ is bounded as soon as it is $\frac{b(t)}{a(t)}$.

Set $z(t):=\frac{b(t)}{a(t)}$ for convenience. If $r-\lambda-\mu \neq 0$ we have

$$
\left\{\begin{array}{l}
\dot{z}(t)=(r-\lambda-\mu) z(t)-\theta, \quad t \in \mathbb{T}^{-}, \\
z(T)=1
\end{array}\right.
$$

whose solution is

$$
z(t)=\frac{\theta}{r-\lambda-\mu}\left[1-\mathrm{e}^{-(r-\lambda-\mu)(T-t)}\right]+\mathrm{e}^{-(r-\lambda-\mu)(T-t)}, \quad t \in \mathbb{T} .
$$

Otherwise, if $r-\lambda-\mu=0$ we obtain

$$
\left\{\begin{array}{l}
\dot{z}(t)=-\theta, \quad t \in \mathbb{T}^{-} \\
z(T)=1
\end{array}\right.
$$

whose solution is

$$
z(t)=1+\theta(T-t), \quad t \in \mathbb{T} .
$$

Observe that either solution (28) or (29) is such that $z(t)>0$, for any $t \in \mathbb{T}$, and so both of them are bounded from below.

Let be $r-\lambda-\mu=0$, then

$$
\max _{t \in \mathbb{T}}\{z(t)\}=z(0)=1+\theta T,
$$

i.e., solution (29) is bounded from above.

Let be $r-\lambda-\mu \neq 0$, then

$$
\begin{gathered}
r-\lambda-\mu=\theta \Rightarrow \max _{t \in \mathbb{T}}\{z(t)\}=1, \\
r-\lambda-\mu>\theta \Rightarrow \max _{t \in \mathbb{T}}\{z(t)\} \leq 1, \\
r-\lambda-\mu<\theta \Rightarrow \max _{t \in \mathbb{T}}\{z(t)\}=\frac{\theta}{r-\lambda-\mu}\left[1-\mathrm{e}^{-(r-\lambda-\mu) T}\right]+\mathrm{e}^{-(r-\lambda-\mu) T},
\end{gathered}
$$

and thus solution (28) is bounded from above, too.

Under conditions (15) Proposition 5.8 holds, i.e., for any initial data $(t, x, h) \in \mathbb{T} \times \Lambda$, the map $\bar{\ell}(t, x, h)$ is bounded.

Therefore, the existence and uniqueness for every $(t, x, h) \in \mathbb{T} \times \Lambda$ is due to the Lipschitz continuity of the maps (19) and so the proof is standard.

\section{Proof of Lemma 11}

The proof comes from standard arguments (see, e.g., Yong \& Zhou, 1999: Theo- 
rem 3.7, p. 241), but we review it for convenience. Let us take $(c(\cdot), \ell(\cdot)) \in \mathcal{V}_{\text {ad }}(t, x, h)$ such that conditions (18) are satisfied. By Lemma 5 the function $v$ defined in (16) is a classical solution to the HJB Equation (12), thus

$$
\begin{aligned}
& \frac{\mathrm{d}}{\mathrm{d} s} v(s, x(s), h(s)) \\
= & v_{t}(s, x(s), h(s))+v_{x}(s, x(s), h(s)) \frac{\mathrm{d}}{\mathrm{d} s} x(s)+v_{h}(s, x(s), h(s)) \frac{\mathrm{d}}{\mathrm{d} s} h(s) \\
= & -\mathcal{H}\left(s, x(s), h(s), v_{x}(s, x(s), h(s)), v_{h}(s, x(s), h(s))\right) \\
& +[w(s) \ell(s)-c(s)+r x(s)] v_{x}(s, x(s), h(s)) \\
& +[\lambda+\mu \ell(s)] v_{h}(s, x(s), h(s)) \\
= & -\mathcal{H}\left(s, x(s), h(s), v_{x}(s, x(s), h(s)), v_{h}(s, x(s), h(s))\right) \\
& +\mathcal{H}_{c v}\left(s, x(s), h(s), v_{x}(s, x(s), h(s)), v_{h}(s, x(s), h(s))\right) \\
& -\mathrm{e}^{-\rho s}[\alpha \ln c(t)+\beta \ln w(1-\ell(t))] .
\end{aligned}
$$

Integrating both sides of the above relation from $t$ up to $T$ we obtain

$$
\begin{aligned}
& \int_{t}^{T} \frac{\mathrm{d}}{\mathrm{d} s} v(s, x(s), h(s)) \mathrm{d} s \\
& =\int_{t}^{T}\left[-\mathcal{H}\left(s, x(s), h(s), v_{x}(s, x(s), h(s)), v_{h}(s, x(s), h(s))\right)\right. \\
& \left.\quad+\mathcal{H}_{c v}\left(s, x(s), h(s), v_{x}(s, x(s), h(s)), v_{h}(s, x(s), h(s))\right)\right] \mathrm{d} s \\
& \quad-\int_{t}^{T} \mathrm{e}^{-\rho s}[\alpha \ln c(t)+\beta \ln w(1-\ell(t))] \mathrm{d} s,
\end{aligned}
$$

i.e.,

$$
\begin{aligned}
& v(t, x, h)-v(T, x(T), h(T)) \\
& =\int_{t}^{T}\left[\mathcal{H}\left(s, x(s), h(s), v_{x}(s, x(s), h(s)), v_{h}(s, x(s), h(s))\right)\right. \\
& \left.\quad-\mathcal{H}_{c v}\left(s, x(s), h(s), v_{x}(s, x(s), h(s)), v_{h}(s, x(s), h(s))\right)\right] \mathrm{d} s \\
& \quad+\int_{t}^{T} \mathrm{e}^{-\rho s}[\alpha \ln c(t)+\beta \ln w(1-\ell(t))] \mathrm{d} s .
\end{aligned}
$$

Recalling that $v(T, x(T), h(T))=\frac{\alpha+\beta}{\rho} \mathrm{e}^{-\rho T} \ln [a(T) x+b(T) h]$, we finally have

$$
\begin{aligned}
v(t, x, h)= & J(t, x, h ; c(\cdot), \ell(\cdot)) \\
& +\int_{t}^{T}\left[\mathcal{H}\left(s, x(s), h(s), v_{x}(s, x(s), h(s)), v_{h}(s, x(s), h(s))\right)\right. \\
& \left.-\mathcal{H}_{c v}\left(s, x(s), h(s), v_{x}(s, x(s), h(s)), v_{h}(s, x(s), h(s))\right)\right] \mathrm{d} s,
\end{aligned}
$$

and the claim is proved.

\section{Proof of Theorem 12 (Verification Theorem)}

By applying Lemma 11 , for any $(t, x, h) \in \mathbb{T} \times \Lambda$, we derive the fundamental identity (24), that we recall for convenience 


$$
\begin{aligned}
& v(t, x, h)=J(t, x, h ; c(\cdot), \ell(\cdot)) \\
& +\int_{t}^{T}\left[\mathcal{H}\left(s, x(s), h(s), v_{x}(s, x(s), h(s)), v_{h}(s, x(s), h(s))\right)\right. \\
& \left.-\mathcal{H}_{c v}\left(s, x(s), h(s), v_{x}(s, x(s), h(s)), v_{h}(s, x(s), h(s)) ; c(\cdot), \ell(\cdot)\right)\right] \mathrm{d} s,
\end{aligned}
$$

The above relation holds for any $(c(\cdot), \ell(\cdot)) \in \mathcal{V}_{\mathrm{ad}}(t, x, h)$ with the corresponding state trajectory $(x(\cdot), h(\cdot))$. Moreover, since $\mathcal{H}(\cdot) \geq \mathcal{H}_{c v}(\cdot ; c, \ell)$, for every $(c, \ell) \in \Gamma$, then $v \geq V$.

Taking into account Proposition 8, Lemma 7, and Lipschitz continuity of the feedback map (19), the feedback strategy defined in (25) is admissible, i.e., $\left(c^{\star}(\cdot), \ell^{\star}(\cdot)\right) \in \mathcal{V}_{\text {ad }}(t, x, h)$. Furthermore, considering Lemma 5 and (19), we see that, at any time $s \in[t, T]$, the feedback strategy (25) maximize the Hamiltonian current value $\mathcal{H}_{c v}$. Thus, in this case we obtain

$$
v(t, x, h)=J\left(t, x, h ; c^{\star}(\cdot), h^{\star}(\cdot)\right) .
$$

Plugging $\left(c^{\star}(\cdot), \ell^{\star}(\cdot)\right) \in \mathcal{V}_{\text {ad }}(t, x, h)$ into the above fundamental identity, we obtain $\mathcal{H}-\mathcal{H}_{c v}=0$, we obtain

$$
V(t, x, h) \geq J\left(t, x, h ; c^{\star}(\cdot), h^{\star}(\cdot)\right)=v(t, x, h) \geq V(t, x, h),
$$

namely, $v(t, x, h)=V(t, x, h)$ and $\left(c^{\star}(\cdot), \ell^{\star}(\cdot)\right)$ defined in (25) is optimal.

The uniqueness of the optimal strategy is consequence of the characterization (25) and of the uniqueness of solutions to the closed loop equation stated in Lemma 9. 